\title{
INFECCIONES ASOCIADAS A LA ATENCIÓN DE SALUD EN EL INSTITUTO NACIONAL MATERNO PERINATAL
}

\author{
Oswaldo M. Gonzales Carrillo', Jesus Pardo Meza², Elizabeth Yañez Alvarado ${ }^{3}$, Priscilla Salazar Barreda
}

\section{RESUMEN}

Objetivos: Determinar las tasas de prevalencia e incidencia de las Infecciones Asociadas a la Atención de Salud (IAAS) en el Instituto Nacional Materno Perinatal (INMP) del 2012 al 2016. Materiales y métodos: Revisamos los casos de IAAS en una cohorte histórica del 2012 al 2016, la definición de caso de IAAS o Infección Intrahospitalaria (IIH) se realizó según lo establecido en la Norma Técnica del MINSA. La prevalencia de casos se estableció comparándolo con el total de egresos hospitalarios en el INMP ocurridos durante el periodo de estudio. Se establecieron las tasas de incidencia promedio para cada tipo de infección, según factor de riesgo de exposición y servicio de ocurrencia. Estas tasas fueron comparadas con las tasas de incidencia nacional establecidas por el Centro Nacional de Epidemiologia, Prevención y Control de Infecciones del MINSA - Perú. Resultados: El número de casos de IAAS en el periodo de estudio fue 1,498 casos. La prevalencia de IAAS fue 1.24\% (1.24 casos del total de egresos hospitalarios). En el Servicio de Neonatología, la Densidad Incidencia de Infección del Tracto Sanguíneo por Catéter Venoso Central fue 5.36 casos por 1000 d.d.e., la Densidad Incidencia de Infección del Tracto Sanguíneo por Catéter Venoso Periférico fue 3.77 casos por 1000 d.d.e. y la Densidad Incidencia de Neumonía Intrahospitalaria por Ventilación Mecánica fue 2.31 casos por 1000 d.d.e.. En el Servicio de Gíneco-Obstetricia, la incidencia acumulada de Endometritis por Parto Vaginal fue $0.23 \%$, la incidencia acumulada de Endometritis por Cesárea fue $0.44 \%$ y la incidencia acumulada de Infecciones de Herida Operatoria por Cesárea fue $1.55 \%$. En la Unidad de Cuidados Intensivos Materno, la Densidad Incidencia de Infecciones Urinarias por Catéter Urinario fue 1.70 casos por 1000 d.d.e., la Densidad Incidencia de las Infecciones por Catéter Venoso Central fue 7.81 casos por 1000 d.d.e. y la Densidad Incidencia de las Neumonías Intrahospitalarias por Ventilación Mecánica fue 14.29 casos por 1000 d.d.e. Las Infecciones del Torrente Sanguíneo por Catéter Venoso Central en la Unidad de Cuidados Intensivos Materno y las Infecciones del Torrente Sanguíneo por Catéter Venoso Periférico en el Servicio de Neonatología están por encima del P90 de rango de valores estándar nacional. Las Neumonías por Ventilación Mecánica en la Unidad de Cuidados Intensivos Materno están por debajo del P90, pero se observa un aumento inusitado de sus tasas en el 2016 en 25.95 casos por 1000 d.d.e. Conclusiones: Aunque es preferible que la evaluación de la prevalencia de IAAS sea puntual, concluimos que la prevalencia de $1.24 \%$ de casos en relación a los egresos hospitalarios, permite un parámetro de evaluación institucional anual. Por su alta incidencia comparado con las tasas nacionales, las infecciones del torrente sanguíneo por catéter venoso central en la unidad de cuidados intensivos materno y las infecciones del torrente sanguíneo por catéter venoso periférico en la unidad de cuidados intensivos e intermedios en el servicio de neonatología requieren acciones de control inmediatos. Los casos de neumonía por ventilación mecánica en la unidad de cuidados intensivos materno también requieren acciones de control por el aumento inusitado en los últimos años.

Palabras Clave: Infección Nosocomial; Infección en la Atención de Salud; Programas de Vigilancia (Fuente DeCS BIREME).

\section{HEALTHCARE ASSOCIATED INFECTIONS IN THE NATIONAL INSTITUTE OF MATERNAL AND PERINATAL HEALTH}

\section{ABSTRACT}

Objective: To determine the prevalence and incidence rates of Healthcare Associated Infections (IAAS) in the National Institute of Maternal and Perinatal Health (INMP) from 2012 to 2016. Materials and methods: We reviewed the cases of IAAS in a historical cohort from 2012 to 2016, the case definition of IAAS or intrahospital Infection (IIH) was performed according to the Technical Standard of MINSA. The prevalence was established comparing it with the total of hospital discharges during the study period. The average incidence rates were established for each type of infection, according to risk factor of exposure and service of occurrence. These rates were compared with the national incidence rates established by the National Center for Epidemiology, Prevention and Control of Infections of MINSA - Peru. Results: The number of IAAS cases in the study period was 1,498 . The prevalence of IAAS was $1.24 \%$ (1.24 cases of total hospital discharges). In the Neonatal Service, Incidence Density of Infection by Central Venous Catheter was 5.36 cases per 1000 d.d.e., Incidence of Infection by Peripheral Venous Catheter was 3.77 cases per 1000 d.d.e.; and Density Incidence of Pneumonia by Mechanical Ventilation was 2.31 cases per 1000. In the Gynaecological-Obstetrics Service, accumulative incidence of Vaginal Endometritis was $0.23 \%$, accumulative incidence of Endometritis by Cesarean section was $0.44 \%$, and accumulated incidence of operative wound infections by Cesarean section was $1.55 \%$. In the Maternal Intensive Care Unit, Incidence Density of Urinary Infections by Urinary Catheter was 1.70 cases per 1000 d.d.e., Incidence Density of infection by Central Venous Catheter was 7.81 cases per 1000 d.d.e.; and Density Incidence of Pneumonia by Mechanical Ventilation was 14.29 cases per 1000 . When comparing institutional rates with national rates, Incidence infection by Central Venous Catheter on the Maternal Intensive Care Unit and Infections by Peripheral Venous Catheter Blood in the Neonatal Service are above the P90 national standard range

Médico Gíneco-Obstetra. Jefe de la Oficina de Epidemiologia y Salud Ambiental. Instituto Nacional Materno Perinatal. Lima - Perú.

2 Médico Cirujano. Área de Investigación Epidemiológica y Análisis Situacional de la Oficina de Epidemiologia y Salud Ambiental del Instituto Nacional Materno Perinatal. Lima - Perú.

3 Licenciada en Enfermería. Área de Vigilancia Epidemiológica de la Oficina de Epidemiologia y Salud Ambiental del Instituto Nacional Materno Perinatal. Lima - Perú.

4 Licenciada en Obstetricia. Área de Vigilancia Epidemiológica de la Oficina de Epidemiologia y Salud Ambiental del Instituto Nacional Materno Perinatal. Lima - Perú. 
of values. Pneumonias by Mechanical Ventilation in Maternal Intensive Care Unit are below P90, but there is an unprecedented increase in their rates in 25.96 cases per 1000 d.d.e. Conclusions: Although the prevalence of IAAS is frecuently performed within 24 hours, we conclude prevalence of $1.24 \%$ in relation to hospital discharges allows an annual institutional evaluation parameter. Due to high incidence compared to the national rates, infection by central venous catheter in maternal intensive care unit and infection by peripheral venous catheter in the intensive and intermediate care unit of the neonatology service require immediate control. Cases of pneumonia by mechanical ventilation in the maternal intensive care unit also require control because unusual increase in recent years.

Key words: Nosocomial infection, Healthcare infection, Surveillance programs (Source: MeSH NLM).

\section{INTRODUCCIÓN}

Las Infecciones Asociadas a la Atención de Salud, antes llamado Infecciones Intrahospitalarias son un indicador que mide la calidad de los servicios prestados. La medida de la calidad de los servicios no solo se basa en los índices de mortalidad y/o morbilidad y el aprovechamiento de los recursos hospitalarios, sino también se toma en cuenta la incidencia de infecciones adquiridas durante la hospitalización. No es permitido que en un hospital o establecimiento de salud el paciente venga a enfermar ${ }^{1}$. Se define como Infección Asociada a la Atención de Salud a aquella condición local o sistémica resultante de una reacción adversa a la presencia de un agente infeccioso o a su toxina(s) que ocurre en un paciente en un escenario de atención de salud (hospitalización o atención ambulatoria) y que no estaba presente en el momento de la admisión, también son consideradas las infecciones que estén relacionadas a una admisión previa. Asimismo incluyen las infecciones ocupacionales contraídas por el personal sanitario. Esta definición reemplazará a otras utilizadas en el país tales como Infección Nosocomial, Infección Intrahospitalaria o Infecciones Asociadas al Cuidado de la Salud (IACS) ${ }^{2,3}$.

La situación de las infecciones intrahospitalarias en las diferentes regiones del mundo fue demostrado por la OMS, quienes realizaron un estudio de prevalencia en 55 hospitales de 14 países representativos de Europa, Mediterráneo Oriental, Asia Sudoriental y el Pacifico Occidental durante el año de 1987, encontrando que un promedio de $8,7 \%$ de los pacientes hospitalizados presentaban infecciones nosocomiales (1.4 millones de personas alrededor del mundo sufren complicaciones por infecciones intrahospitalarias). La máxima frecuencia de infecciones se reportó en el Mediterráneo Oriental y Asia Sudoriental (11.8 y $10 \%$ respectivamente), seguido de las regiones de Europa y del Pacífico Occidental (7.7 y $9,0 \%$ respectivamente). Las infecciones más frecuentes son las de heridas quirúrgicas, las vías urinarias y las vías respiratorias inferiores ${ }^{4}$.
En las Unidades de Cuidados Intensivos, las cifras son más alarmantes y varían entre países en vías de desarrollo y países desarrollados, observándose en los primeros una densidad incidencia de $47.9 \times 1000$ días de exposición a los dispositivos aplicados y en países desarrollados una densidad incidencia de 13.6 por 1000 días de exposición. Las tasas de infecciones asociadas a dispositivos son mucho más altas en países latinoamericanos comparadas con las de USA, debido a programas de control aún muy deficientes y falta de acreditación hospitalaria, además de recursos limitados para la ejecución de políticas de prevención ${ }^{5}$.

Los costos estimados por estas infecciones también son significativos. En los Estados Unidos se estimó que por este tipo de infecciones existía un costo de 4 mil millones de dólares al año (estimado en el año 1985), asociados a la estancia prolongada y el costo de tratamiento. En las Américas estos costos han sido estimados aisladamente sobre todo en las unidades de cuidados intensivos, así tenemos que en 2 hospitales de Argentina (2006) se estimaron por ejemplo $\$ 1 ' 233,751$ y $\$ 1 ' 741,872$ al año, similares cifras fueron observadas en hospitales de otros países vecinos ${ }^{6}$.

La Organización Panamericana de la Salud describe que más de 1,4 millones de personas en el mundo contraen infecciones en el hospital, además recalca que entre el $5 \%$ y el $10 \%$ de los pacientes que ingresan a hospitales modernos de países desarrollados contraerán una o más infecciones, sostiene también que el riesgo de IAAS en los países en desarrollo es de 2 a 20 veces mayor que en los países desarrollados y que se calcula que las infecciones relacionadas con la atención sanitaria en Inglaterra generan un costo de 1.000 millones de libras por año. En los Estados Unidos la cifra es de entre 4.500 millones y 5.700 millones de US\$ y en México el costo anual se aproxima a los 1.500 millones de dólares ${ }^{7,8,9}$.

En el Perú, el panorama es similar. Según datos presentados en el Boletín Epidemiológico por el Centro Nacional de Epidemiologia, Prevención y Control de Enfermedades del MINSA, el total de Infecciones Asociadas a la Atención de Salud a nivel nacional para el periodo 2012 - 2014 fueron 10,938 casos, siendo la infección por herida operatoria la infección más frecuente (2933 casos), seguido por las neumonías asociadas a ventilación mecánica (2,219 casos), Infecciones del Tracto Urinario asociado a Catéter Urinario Permanente $(2,068 \text { casos })^{9,10}$.

En el INMP, la vigilancia de IAAS se reporta desde el año 1997 y a través de los años han mejorado las estrategias de prevención y control de los mismos observándose una disminución significativa de las cifras a la actualidad. Sin embargo, y ante la creciente complejidad de los casos observados, existe un riesgo inherente para una Infección Nosocomial que es necesario evaluar y analizar para mejorar la calidad de atención a los pacientes que 
ingresan a la institución, sobre todo los pacientes que ingresan a cuidados intensivos maternos y neonatales ${ }^{11}$.

Ante esta situación la Oficina de Epidemiologia y Salud Ambiental propone un estudio de prevalencia e incidencia de casos de Infecciones Asociadas a la Atención de Salud ocurridos durante los últimos 5 años (Enero del 2012 a Diciembre del 2016), con el objetivo de conocer el comportamiento de estas infecciones y compararnos con las tasas nacionales para la toma inmediata de acciones.

\section{MATERIAL Y MÉTODOS}

Este es un estudio de incidencia, de una cohorte histórica de casos de Infecciones Asociadas a la Atención de Salud ocurridas en el Instituto Nacional Materno Perinatal. Revisamos la base de datos que maneja la Oficina de Epidemiologia y Salud Ambiental, con la que reportan los casos de Infecciones Asociadas a la Atención de Salud en forma mensual al Centro Nacional de Epidemiologia, Prevención y Control de Enfermedades - MINSA, del 2012 al 2016. Infección Asociada a la Atención de Salud fue definida según los Lineamientos establecidos en el Documento Técnico: Lineamientos para la Vigilancia, Prevención, y Control de las Infecciones Asociadas a la Atención de Salud, documento que entró en vigencia el 16 de marzo del 2015, con Resolución Ministerial 1682015/MINSA. Sin embargo las definiciones de caso para la vigilancia epidemiológica están establecidas en el Manual de Epidemiologia Aplicada a la Vigilancia de las Infecciones Intrahospitalarias, emitida en Julio del 2004 por la Oficina General de Epidemiologia y es con la que cuenta la Oficina de Epidemiologia del INMP para realizar la búsqueda activa de casos.

Los casos fueron identificados en forma prospectiva y con vigilancia activa y dirigida en los servicios de hospitalización del Instituto, definidos como Servicios de Gíneco-Obstetricia (A, B, C, D y E, Servicio Bajo Tarifario Diferenciado), la Unidad de Cuidados Intensivos Materno, y el Servicio de Neonatología (Unidad de Cuidados Intensivos e Intermedios Neonatales). La búsqueda activa está dirigida a definir y vigilar casos de infecciones intrahospitalaria en Infecciones de Herida Operatoria por Cesárea, Endometritis Puerperal por Parto Vaginal, Endometritis Puerperal por Parto por Cesárea en los Servicios de Gineco-Obstetricia, Infección del Torrente Sanguíneo por Catéter Venoso Periférico en la Unidad de Cuidados Intensivos e Intermedios Neonatales, Infección del Torrente Sanguíneo por Catéter Venoso Central en la Unidad de Cuidados Intensivos Materno y en la Unidad de Cuidados Intensivos e Intermedios Neonatales, Neumonía por Ventilación Mecánica en la Unidad de Cuidados Intensivos Materno y en la Unidad de Cuidados Intensivos e Intermedios Neonatales y finalmente Infección del Tracto Urinario por Catéter Urinario Permanente en la Unidad de Cuidados Intensivos Materno.
Se elaboraron gráficas para describir el número de casos encontrados en cada año, y la tendencia por años, así como la prevalencia de casos en los cinco años estudiados definido como el número de casos de IAAS en los 5 años estudiados comparado con el total de egresos hospitalarios ocurridos en los mismos.

Se definen las tasas de incidencias de casos por servicios de ocurrencia, tipo de infección y factor de exposición en los 5 años de estudio. Estas tasas fueron comparadas con las tasas nacionales obtenidas por el Centro Nacional de Epidemiologia, Prevención y Control de Enfermedades - MINSA, evaluados para los años 2013 - 2014, para observar la magnitud del problema de las IAAS en la institución y que tipo de infección es la que debemos abordar con urgencia.

\section{RESULTADOS}

Los casos de Infecciones Asociadas a la Atención de Salud (IAAS), en el periodo 2012 al 2016, fueron en total 1498 casos notificados, con un promedio de 156,414 días/ cama vigilados y 89,023 procedimiento realizados, además teniendo en cuenta que, los egresos hospitalarios del Instituto desde el año 2012 al 2016 han sido 121,070 pacientes, podemos definir que la prevalencia de casos de IAAS en relación con el total de egresos hospitalarios durante los 5 años estudiados es $1.24 \%$ (es decir existe 1.24 casos de IAAS por cada 100 pacientes que egresan de la institución). La distribución de casos y la prevalencia por años lo observamos en la gráfica 1.

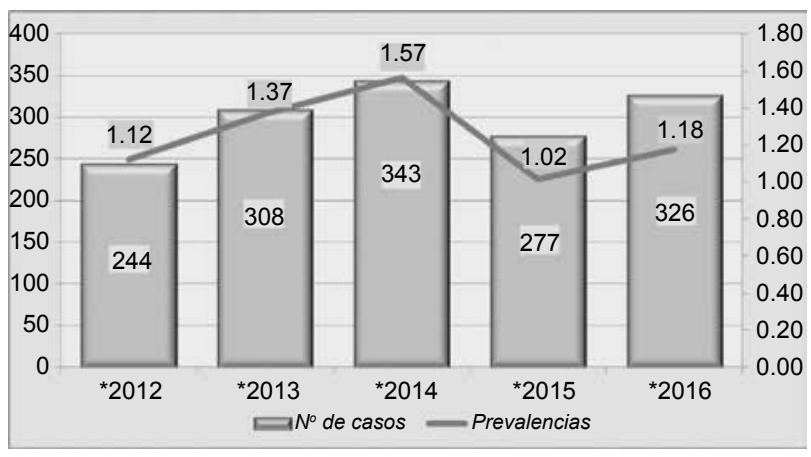

Gráfico 1. Número de Casos de IAAS y Prevalencia de Casos por Egresos Hospitalarios. 2012 al 2016 - INMP

El total de casos notificados en el Servicio de GínecoObstetricia (incluyendo servicios de hospitalización y la Unidad de Cuidados Intensivos Materno) fueron 928 casos y en el Servicio de Neonatología (que incluye la Unidad de Cuidados Intensivos y Unidad de Cuidados Intermedios) fueron 570 casos. Se observa en la siguiente gráfica 2 la distribución de casos por servicios desde los años 2012 al 2016. 


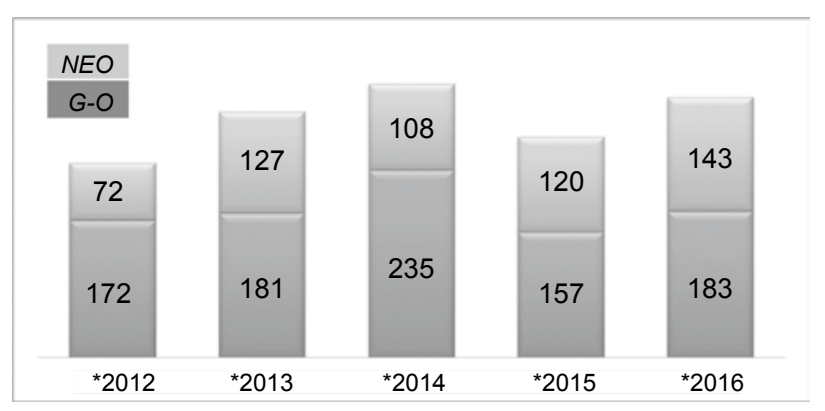

Gráfico 2. Número de Casos de IAAS por Servicio. INMP 2012 - 2016

Al analizar el número de casos de infecciones según exposición al factor de riesgo en los 5 años estudiados, observamos que la infección más frecuente es la Infección de Herida Operatoria por Cesárea en el Servicio de GínecoObstetricia con 591 casos (39.5\% del total de casos), seguido por la Infección del Torrente Sanguíneo por Catéter Venoso Periférico en el Servicio de Neonatología con 378 casos (25.2\%), sigue la Endometritis por Cesárea en GínecoObstetricia con 169 casos (11.3\%), la Infección del Torrente Sanguíneo por Catéter Venoso Central en Neonatología con 148 casos (9.9\%), la Endometritis por Parto Vaginal en Gíneco-Obstetricia con 116 casos (7.7\%), Neumonía por Ventilación Mecánica en Neonatología con 44 casos (2.9\%), Neumonía por Ventilación Mecánica en la Unidad de Cuidados Intensivos Materno con 24 casos (1.6\%), las Infecciones del Torrente Sanguíneo por Catéter Venoso Central en la Unidad de Cuidados Intensivos Materno con 19 casos $(1.3 \%)$ y finalmente las Infecciones del Tracto Urinario por Catéter Urinario Permanente en la Unidad de Cuidados Intensivos Materno con 9 casos (0.6\%) Gráfica 3.

Si analizamos la tendencia del número de casos de infecciones a través de los años observamos que, en el Servicio de Neonatología la infección que más casos presentó es la Infección del Tracto Sanguíneo por Catéter Venoso Periférico, la cual mantiene una tendencia alta en

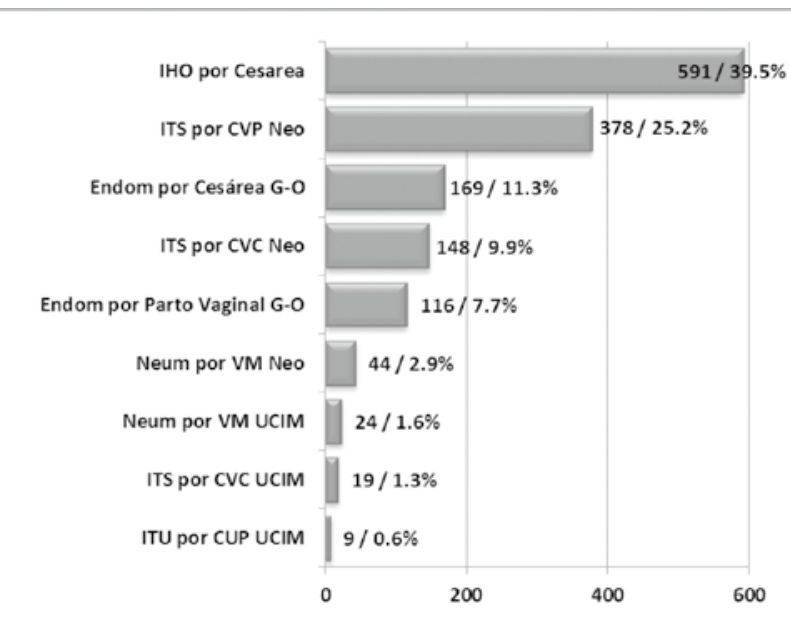

Gráfica 3. Número de Casos de IAAS según Exposición al Factor de Riesgo. INMP 2012 - 2014 comparación con el resto de infecciones presentadas en el servicio. Sin embargo, observamos que este último año el número de casos de Infecciones del Tracto Sanguíneo por Catéter Venoso Central tuvo un aumento significativo en relación a los años anteriores. La Neumonía por Ventilación Mecánica ha tenido una tendencia a la baja.

En el Servicio de Gíneco-Obstetricia observamos que la infección más frecuente son las Infecciones de Herida Operatoria por Cesárea, la curva siempre se ha mantenido en cifras altas a través de los años, a comparación de los casos de Endometritis por Cesárea y por Parto Vaginal.

En la Unidad de Cuidados Intensivos Materno, las Infecciones del Tracto Urinario han disminuido a través de los años, sin presencia de casos para el 2016; sin embargo las Infecciones del Tracto Sanguíneo por Catéter Venoso Central ha seguido la tendencia a ser cada vez mayor y lo que ha llamado mucho la atención es la subida en el número de casos de Neumonias Intrahospitalarias por Ventilación Mecánica. Estas tendencias se muestras en la Gráfica 4.

\section{Densidad Incidencia de Infecciones Asociadas en la Atención de Salud en Neonatología (Unidad de Cuidados Intensivos y Unidad de Cuidados Intermedios)}

Cuando evaluamos las tasas de incidencia de casos en el Servicio de Neonatología observamos que la Densidad Incidencia promedio de Infección del Tracto Sanguíneo por Catéter Venoso Central en los 5 años es 5.36 casos por 1000 días de exposición al dispositivo (d.d.e.), la Densidad Incidencia de Infección del Tracto Sanguíneo por Catéter Venoso Periférico en los 5 años es 3.77 casos por 1000 d.d.e. y finalmente la Densidad Incidencia de

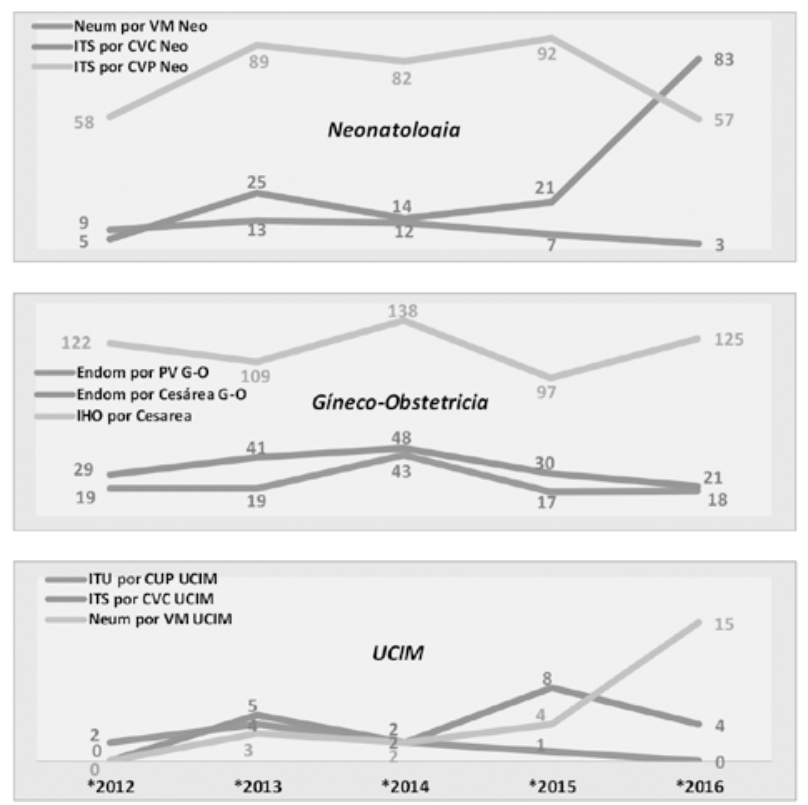

Gráfica 4. Tendencia por Años en el Número de Casos de Infecciones por Factor de Riesgo Asociado presentado en cada Servicio. INMP 2012 - 2016 
Tabla 1. Densidad Incidencia de las IAAS en Neonatología. INMP 2012 - 2016

\begin{tabular}{|c|c|c|c|c|c|c|c|c|c|c|c|c|}
\hline \multirow[b]{2}{*}{ Año } & \multicolumn{4}{|c|}{ Cáteter Venoso Central (CVC) } & \multicolumn{4}{|c|}{ Cáteter Venoso Periférico (CVP) } & \multicolumn{4}{|c|}{ Ventilación Mecánica (VM) } \\
\hline & $\begin{array}{l}N^{\circ} \text { días de } \\
\text { exposición }\end{array}$ & $\begin{array}{c}\mathrm{N}^{\circ} \\
\text { pacientes } \\
\text { vigilados }\end{array}$ & $\begin{array}{c}\mathrm{N}^{\circ} \text { ITS } \\
\text { asociados } \\
\text { a CVC }\end{array}$ & $\begin{array}{c}\text { Densidad } \\
\text { Incidencia }\end{array}$ & $\begin{array}{l}N^{\circ} \text { días de } \\
\text { exposición }\end{array}$ & \begin{tabular}{c}
\multicolumn{1}{c}{$\mathrm{N}^{\circ}$} \\
pacientes \\
vigilados
\end{tabular} & $\begin{array}{c}\mathrm{N}^{\circ} \text { ITS } \\
\text { asociados } \\
\text { a CVP }\end{array}$ & $\begin{array}{l}\text { Densidad } \\
\text { Incidencia }\end{array}$ & $\begin{array}{l}N^{0} \text { días de } \\
\text { exposición }\end{array}$ & $\begin{array}{c}\mathrm{N}^{\circ} \\
\text { pacientes } \\
\text { vigilados }\end{array}$ & $\begin{array}{c}\mathrm{N}^{\circ} \text { Neum. } \\
\text { asociado } \\
\text { a VM }\end{array}$ & $\begin{array}{c}\text { Densidad } \\
\text { Incidencia }\end{array}$ \\
\hline 2012 & 4181 & 607 & 5 & 1.20 & 18671 & 4564 & 58 & 3.11 & 3862 & 543 & 9 & 2.33 \\
\hline 2013 & 4367 & 600 & 25 & 5.72 & 18066 & 3961 & 89 & 4.93 & 2326 & 353 & 13 & 5.59 \\
\hline 2014 & 4381 & 564 & 14 & 3.20 & 16571 & 3981 & 82 & 4.95 & 2560 & 396 & 12 & 4.69 \\
\hline 2015 & 4966 & 585 & 21 & 4.23 & 20530 & 3665 & 92 & 4.48 & 3438 & 443 & 7 & 2.04 \\
\hline 2016 & 9735 & 1510 & 83 & 8.53 & 26502 & 4410 & 57 & 2.15 & 6848 & 1003 & 3 & 0.44 \\
\hline Total & 27630 & 3866 & 148 & 5.36 & 100340 & 20581 & 378 & 3.77 & 19034 & 2738 & 44 & 2.31 \\
\hline
\end{tabular}

Neumonía Intrahospitalaria por Ventilación Mecánica en los 5 años es 2.31 casos por 1000 d.d.e. al dispositivo. La distribución de las tasas de incidencia por años lo observamos en la Tabla 1.

La tendencia de las tasas de Densidad Incidencia a través de los años 2012 - 2016, se observa que en los casos de infecciones por Catéter Venoso Periférico y por Ventilación Mecánica, las incidencias tienden a la baja; sin embargo, la Incidencia de infecciones por Catéter Venoso Central ha tenido un aumento importante este último año, como se observa en la Gráfica 5.

Analizamos también, las tasas de incidencia de casos en relación al peso del Recién Nacido, y observamos que la mayor incidencia de infecciones ocurre cuando el peso del recién nacido es menor de 1500 gr, además podemos observar que la infección de mayor incidencia en este grupo de neonatos (menores de $1500 \mathrm{gr}$ ) es la causada por Catéter Venoso Periférico, mientras que en mayores de $1500 \mathrm{gr}$ la principal causa de infecciones es por Catéter Venoso Central. Tabla 2.

\section{Incidencia de Infecciones Asociadas en la Atención de} Salud en los Servicios de Gíneco Obstetricia

En el Servicio de Gíneco-Obstetricia se realiza la vigilancia de IAAS en 3 factores de riesgo: Endometritis por Parto Vaginal, Endometritis por Parto Cesárea e Infecciones

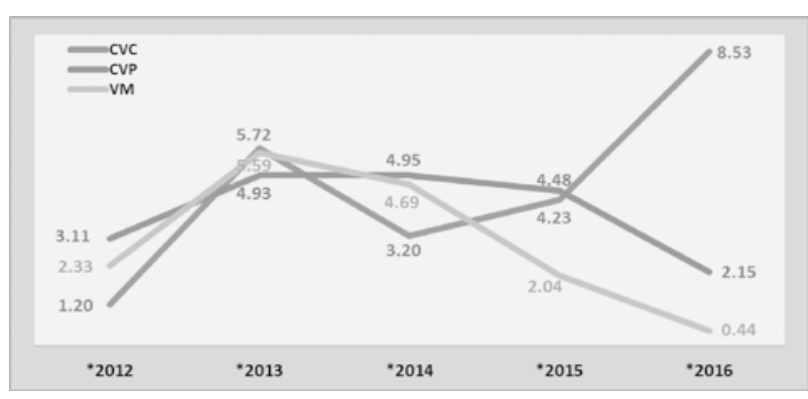

Gráfica 5. Tendencia por Años en la Incidencia de Casos de IAAS por Factor de Riesgo en Neonatología. INMP 2012 - 2016

Tabla 2. Incidencia de IAAS según el peso del Recién Nacido. INMP 2012 - 2016

\begin{tabular}{lccc}
\hline \multicolumn{1}{c}{ Factor de Riesgo } & $\mathbf{< 1 5 0 0} \mathbf{~ g r}$ & $\mathbf{1 5 0 0} \mathbf{- 2 5 0 0} \mathbf{~ g r}$ & $\boldsymbol{>} \mathbf{2 5 0 0} \mathbf{~ g r}$ \\
\hline Catéter Venoso Central & 6.37 & 5.24 & 1.87 \\
Catéter Venoso Periférico & 9.42 & 3.21 & 1.68 \\
Ventilación Mecánica & 2.75 & 2.38 & 1.18 \\
\hline
\end{tabular}

de Herida Operatoria por Cesárea. En los últimos 5 años observamos que la incidencia acumulada promedio de Endometritis por Parto Vaginal es 0.23 casos por cada 100 partos vaginales, la incidencia de Endometritis por Cesárea es 0.44 casos por cada 100 cesáreas y la incidencia de Infecciones de Herida Operatoria por Cesárea es 1.55

Tabla 3. Densidad Incidencia de las IAAS en los Servicios de Gíneco- Obstetricia. INMP 2012 - 2016

\begin{tabular}{|c|c|c|c|c|c|c|c|c|c|}
\hline \multirow[b]{2}{*}{ Año } & \multicolumn{3}{|c|}{ Endometritis por Parto Vaginal } & \multicolumn{3}{|c|}{ Endometritis por Cesárea } & \multicolumn{3}{|c|}{ Inf. de Her. Oper. por Cesarea } \\
\hline & \begin{tabular}{c}
\multicolumn{1}{c}{$\mathrm{N}^{\circ}$ de } \\
Pacientes \\
vigilados
\end{tabular} & $\begin{array}{l}\mathrm{N}^{\circ} \text { Casos de } \\
\text { Endometritis }\end{array}$ & $\begin{array}{l}\text { Incidencia } \\
\text { Acumulada }\end{array}$ & $\begin{array}{l}N^{\circ} \text { de Pacientes } \\
\text { vigilados }\end{array}$ & $\begin{array}{l}\mathrm{N}^{\circ} \text { Casos de } \\
\text { Endometritis }\end{array}$ & $\begin{array}{l}\text { Incidencia } \\
\text { Acumulada }\end{array}$ & $\begin{array}{c}\mathrm{N}^{\circ} \text { de } \\
\text { Pacientes } \\
\text { vigilados }\end{array}$ & $\mathrm{N}^{\circ}$ de IHO & $\begin{array}{l}\text { Incidencia } \\
\text { Acumulada }\end{array}$ \\
\hline 2012 & 7964 & 19 & 0.24 & 5459 & 29 & 0.53 & 5459 & 122 & 2.23 \\
\hline 2013 & 8959 & 19 & 0.21 & 6655 & 41 & 0.62 & 6655 & 109 & 1.64 \\
\hline 2014 & 10792 & 43 & 0.40 & 7280 & 48 & 0.66 & 7280 & 138 & 1.90 \\
\hline 2015 & 11441 & 17 & 0.15 & 8827 & 30 & 0.34 & 8827 & 97 & 1.10 \\
\hline 2016 & 11802 & 18 & 0.15 & 9844 & 21 & 0.21 & 9844 & 125 & 1.27 \\
\hline Total & 50958 & 116 & 0.23 & 38065 & 169 & 0.44 & 38065 & 591 & 1.55 \\
\hline
\end{tabular}


casos por cada 100 cesáreas; esta última es la incidencia más alta encontrada en este servicio (Tabla 3).

La tendencia de las tasas de incidencia acumulada por años, muestra cifras que van a la disminución, excepto en las infecciones de Herida Operatoria que aunque la tendencia también es a disminuir, su disminución es fluctuante y mantiene valores por encima de las otras infecciones. Gráfica 6.

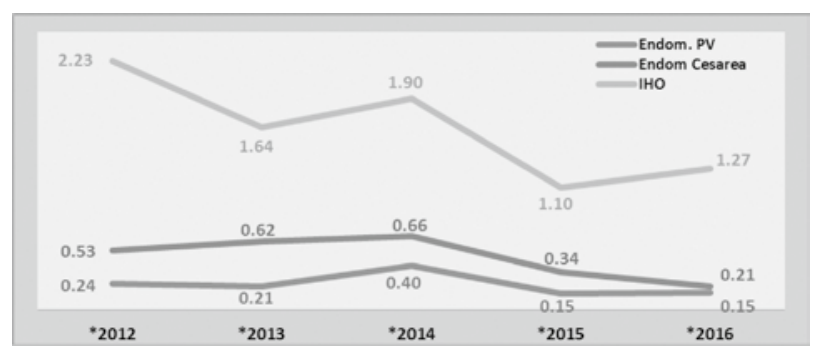

Gráfica 6. Tendencia por Años en la Incidencia de Casos de IAAS por Factor de Riesgo en Gíneco-Obstetricia. INMP 2012 - 2016

\section{Incidencia de Infecciones Asociadas en la Atención de Salud en la Unidad de Cuidados Intensivos Materno}

En la Unidad de Cuidados Intensivos Materno tenemos Infecciones Urinarias por Catéter Urinario con una Densidad Incidencia Promedio en los 5 años estudiados de 1.70 casos por 1000 d.d.e., las Infecciones por Catéter Venoso Central muestra una Densidad Incidencia Promedio de 7.81 casos por 1000 d.d.e. y finalmente las Neumonias Intrahospitalarias por Ventilación Mecánica muestra una Densidad Incidencia Promedio de 14.29 casos por 1000 . Tabla 4.

La tendencia en los últimos 5 años de Densidad Incidencia según tipo de infección en la Unidad de Cuidados Intensivos Materno, muestra que mientras las Infecciones del Tracto Urinario de forma más marcada que las Infecciones del Tracto Sanguíneo por Catéter Venoso Central tienen una disminución en sus valores, la Neumonía Intrahospitalaria ha tenido un ascenso significativo en su incidencia. Gráfica 7.

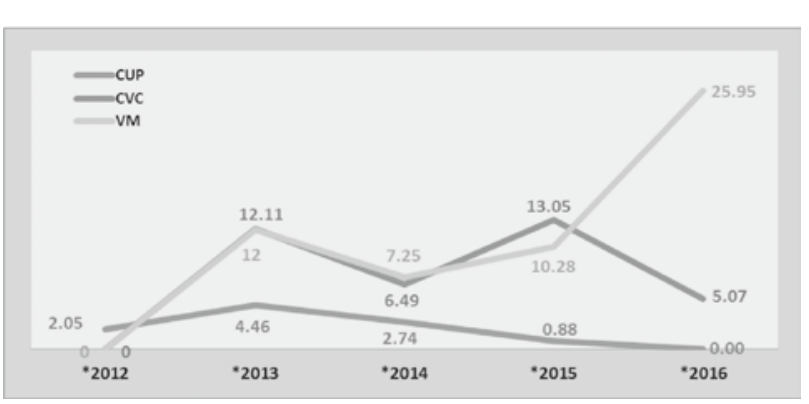

Gráfica 7. Tendencia por Años en la Incidencia de Casos de IAAS por Factor de Riesgo en la Unidad de Cuidados Intensivos Materno. INMP 2012 - 2016

Incidencia de Infecciones Asociadas en la Atención de Salud Promedio Institucional desde el 2012 - 2016.

Las tasas de incidencia promedio institucional, por tipo de atención, factor de riesgo asociado y según el servicio de atención en los últimos 5 años (2012 - 2016), se resumen en la tabla 5 , en ella se observa que las tasas más altas encontradas son las que se refieren a las neumonías intrahospitalarias por ventilación mecánica en la Unidad de Cuidados Intensivos Materno (14.29 x 1000 d.d.e.), las infecciones del torrente sanguíneo por catéter venoso central en la Unidad de Cuidados Intensivos Materno (7.81 x 1000 d.d.e.), las infecciones del torrente sanguíneo

Tabla 5. Tasas de Incidencia Promedio 2012 - 2016 de las IAAS a Nivel Institucional, por Tipo de Infección, Factor de Riesgo y Servicio de Atención

\begin{tabular}{cccc}
\hline $\begin{array}{c}\text { Tipo de } \\
\text { Infección }\end{array}$ & $\begin{array}{c}\text { Factor de } \\
\text { Riesgo }\end{array}$ & Servicio & $\begin{array}{c}\text { Tasa de } \\
\text { Incidencia INMP } \\
\mathbf{( 2 0 1 2}-\mathbf{2 0 1 6})\end{array}$ \\
\hline Neumonia & $\begin{array}{c}\text { Ventilación } \\
\text { Mecanica }\end{array}$ & $\begin{array}{c}\text { Neonatologia } \\
\text { UCl Adultos }\end{array}$ & $\begin{array}{c}2.31 \\
14.29\end{array}$ \\
\hline $\begin{array}{c}\text { Infección } \\
\text { del Torrente } \\
\text { Sanguineo }\end{array}$ & $\begin{array}{c}\text { Cateter Venoso } \\
\text { Central }\end{array}$ & Neonatologia & 5.36 \\
\cline { 2 - 4 } & Cateter Venoso & UCl Adultos & 7.81 \\
\hline Endoriférico & Neonatologia & 3.77 \\
\hline $\begin{array}{c}\text { Infección de Herida } \\
\text { Operatoria }\end{array}$ & Parto Vaginal & G-O & 0.23 \\
\hline $\begin{array}{c}\text { Infección del Tracto } \\
\text { Urinario }\end{array}$ & Parto Cesarea & G-O & 0.44 \\
\hline
\end{tabular}

Tabla 4. Densidad Incidencia de las IAAS en la Unidad de Cuidados Intensivos Maternos. INMP 2012 - 2016

\begin{tabular}{|c|c|c|c|c|c|c|c|c|c|c|c|c|}
\hline \multirow[b]{2}{*}{ Año } & \multicolumn{4}{|c|}{ Cáteter Urinario Permanente (CUP) } & \multicolumn{4}{|c|}{ Cáteter Venoso Central (CVC) } & \multicolumn{4}{|c|}{ Ventilación Mecánica (VM) } \\
\hline & $\begin{array}{l}N^{0} \text { días de } \\
\text { exposición }\end{array}$ & $\begin{array}{l}N^{\circ} \text { pacientes } \\
\text { vigilados }\end{array}$ & $\begin{array}{c}\mathrm{N}^{\circ} \text { ITU } \\
\text { asociados } \\
\text { a CUP }\end{array}$ & $\begin{array}{l}\text { Densidad } \\
\text { Incidencia }\end{array}$ & $\begin{array}{l}N^{\circ} \text { días de } \\
\text { exposición }\end{array}$ & \begin{tabular}{l}
\multicolumn{1}{c}{$\mathrm{N}^{\circ}$} \\
pacientes \\
vigilados
\end{tabular} & $\begin{array}{c}N^{\circ} \text { ITS } \\
\text { asociados } \\
\text { a CVC }\end{array}$ & $\begin{array}{c}\text { Tasa de } \\
\text { ITS } \times 1000\end{array}$ & $\begin{array}{l}N^{\circ} \text { días de } \\
\text { exposición }\end{array}$ & \begin{tabular}{l}
\multicolumn{1}{c}{$\mathrm{N}^{\circ}$} \\
pacientes \\
vigilados
\end{tabular} & $\begin{array}{c}\mathbf{N}^{\circ} \\
\text { Neumonias } \\
\text { asociadas } \\
\text { a VM }\end{array}$ & $\begin{array}{c}\text { Tasa de } \\
\text { ITS x } \\
1000\end{array}$ \\
\hline 2012 & 977 & 264 & 2 & 2.05 & 311 & 81 & 0 & 0 & 186 & 58 & 0 & 0 \\
\hline 2013 & 896 & 219 & 4 & 4.46 & 413 & 71 & 5 & 12.11 & 250 & 50 & 3 & 12 \\
\hline 2014 & 731 & 165 & 2 & 2.74 & 308 & 62 & 2 & 6.49 & 276 & 61 & 2 & 7.25 \\
\hline 2015 & 1142 & 282 & 1 & 0.88 & 613 & 105 & 8 & 13.05 & 389 & 78 & 4 & 10.28 \\
\hline 2016 & 1551 & 426 & 0 & 0 & 789 & 136 & 4 & 5.07 & 578 & 88 & 15 & 25.95 \\
\hline Total & 5297 & 1356 & 9 & 1.70 & 2434 & 455 & 19 & 7.81 & 1679 & 335 & 24 & 14.29 \\
\hline
\end{tabular}


por catéter venoso central en la Unidad de Cuidados Intensivos e Intermedios en Neonatología (5.36 x 1000 d.d.e.) y finalmente las infecciones del torrente sanguíneo por catéter venoso periférico en la Unidad de Cuidados Intensivos e Intermedios en Neonatología (3.77 x 1000 d.d.e.).

\section{DISCUSIÓN}

Las Infecciones Asociadas a la Atención de Salud o las adquiridas durante la asistencia sanitaria son, en su mayoría, prevenibles de eventos adversos graves en pacientes hospitalizados. Estas infecciones ocasionan efectos y a veces discapacidades en la vida de los pacientes, deterioran la imagen de los equipos asistenciales, de los hospitales y del sistema sanitario, y causan un impacto enorme en la economía de un país, poniendo a prueba la sostenibilidad de los programas de salud ${ }^{12}$.

Aunque cada año se realiza Estudios de Prevalencia Puntual de Infecciones Intrahospitalarias, en este estudio hemos querido tener una cifra de Prevalencia que nos pueda brindar información en el número de casos de pacientes que pueden sufrir esta adversidad en relación al total de pacientes que son atendidas en la institución y que egresan de la misma (egresos hospitalarios). Así, obtuvimos una prevalencia calculada en los 5 años aplicados en este estudio de $1.24 \%$ (1.24 casos de infecciones por cada paciente que ha sido atendida y han salido de la institución como egreso hospitalario en condición de alta), teniendo en cuenta que nuestra institución es de nivel III-2 y es preferentemente de atención materno perinatal. Los estudios de Prevalencia Puntuales realizadas en el Perú y en otros países, determinan el número de casos de IAAS en un día programado para el estudio del total de pacientes hospitalizados en ese mismo día. La prevalencia puntual en el país para el año 2015 fue $3.6 \%{ }^{13}$. Si bien no podemos comparar esta cifra de prevalencia puntual con la obtenida en nuestro estudio con el total de egresos hospitalarios, ambos datos son importantes para tener un parámetro establecido de monitoreo de casos en nuestra institución. Igualmente es importante la tendencia en el número absoluto de casos; tenemos así, que las Infecciones de Herida Operatoria son las infecciones más frecuentes en el INMP, lo que no se observa, en las tasas de incidencia en este tipo de infección. Por ello, la evaluación de la frecuencia de infecciones en números absolutos es importante, porque como hospital eminentemente obstétrico, el cuidado de las infecciones de herida operatoria por cesárea es primordial y permite cuestionar el cuidado que estamos brindando para prevenir este tipo de infección.

Quispe Z. reportó en el Boletín Epidemiológico $N^{\circ} 38$ del Centro Nacional de Epidemiología, Prevención y Control de Enfermedades - MINSA, los indicadores de vigilancia epidemiológica de las infecciones intrahospitalarias para los años 2013 - 2014 a nivel nacional. Se presentan en este estudio las tasas de incidencia según el tipo de infección, el factor de riesgo asociado y el servicio hospitalario. Una primera tabla definía las tasas de incidencia general (de todos los establecimientos de salud notificantes) y presentan valores por percentiles que permite saber si estamos por debajo del percentil 25 o por encima del percentil 90. La primera tabla entonces nos permite comparar las tasas del establecimiento de salud con las tasas de referencia a nivel nacional para determinar si están por debajo o encima del valor establecido; asimismo, ubicar la tasa en el percentil según corresponda a fin de identificar valores extremos; así, por ejemplo: si la tasa se ubica por debajo del percentil 25 , indica que puede existir un sub-registro en la vigilancia de las infecciones nosocomiales o que la duración o frecuencia del factor de riesgo es mínimo o escaso; y por el contrario, si se ubica dentro o más allá del percentil 90 , indicará que tiene un comportamiento atípico y puede ser un problema necesario de abordar con prioridad. Si alguna de las tasas institucionales superan a las tasas nacionales y categóricas se debe priorizar en la programación, ejecución de medidas de intervención y documentar la realización e impacto de éstas y lograr comparaciones en valores extremos que permitan tomar acciones. La segunda tabla, muestra valores de las tasas de incidencia nacional por categoría o nivel de establecimiento de salud (establecimientos I-4 hasta establecimientos III-2), lo que permite compararnos con valores de incidencia con otros establecimientos del mismo nivel que el nuestro ${ }^{10}$.

En la tabla 6, comparamos las tasas de incidencia nacional y las tasas de incidencia para Hospitales III-2 como el nuestro, con las tasas promedio obtenidas en nuestra institución en los 5 años estudiados. Además evaluamos nuestros resultados en una columna en la cual colocamos en que percentil se encuentra la tasa encontrada en nuestra institución según la tasa de incidencia nacional obtenida por Centro Nacional de Epidemiología, Prevención y Control de Enfermedades.

Cuando comparamos las tasas obtenidas en el instituto con las tasas referenciales para hospitales nivel III2 , observamos que la tasa que sobrepasa los valores promedio son las infecciones del torrente sanguíneo por catéter venoso central en la Unidad de Cuidados Intensivos Materno (7.81 x 1000 d.d.e. en el instituto contra $3.04 \times 1000$ d.d.e. en el promedio para hospitales nivel III-2), las demás tasas institucionales se encuentran por debajo de los valores promedio para hospitales nivel III-2. Este resultado obliga a la institución, tomar medidas de control para este tipo de infección como planes de intervención u otros que permitan disminuir la carga de infecciones por este factor de riesgo.

Al comparar las tasas de incidencia institucionales promedio en los 5 años de estudio con las tasas obtenidas a nivel nacional, observamos que las tasas son más altas en varios tipos de infecciones como por ejemplo la neumonía 
Tabla 6. Tasas de Incidencia de las IAAS en el INMP comparado con las Tasas Nacionales. INMP 2012 - 2016

\begin{tabular}{|c|c|c|c|c|c|c|c|c|c|c|}
\hline $\begin{array}{l}\text { Tipo de } \\
\text { Infección }\end{array}$ & $\begin{array}{c}\text { Factor de } \\
\text { Riesgo }\end{array}$ & Servicio & $\begin{array}{c}\text { Tasa de } \\
\text { Incidencia INMP } \\
(2012-2016)\end{array}$ & $\begin{array}{c}\text { Tasa Incidencia } \\
\text { Nivel III-2 } \\
(2013-2014)\end{array}$ & $\begin{array}{l}\text { Tasa de } \\
\text { Incidencia } \\
\text { Nacional }\end{array}$ & P25 & P50 & P75 & P90 & Percentil \\
\hline \multirow{2}{*}{ Neumonia } & \multirow{2}{*}{$\begin{array}{l}\text { Ventilación } \\
\text { Mecanica }\end{array}$} & Neonatologia & 2.31 & 5.09 & 4.74 & 0.96 & 2.62 & 6.73 & 11.95 & P25 - P50 \\
\hline & & UCI Adultos & 14.29 & 15.93 & 11.88 & 3.25 & 6.69 & 14.40 & 20.18 & P50 - P75 \\
\hline \multirow{3}{*}{$\begin{array}{l}\text { Infección } \\
\text { del Torrente } \\
\text { Sanguineo }\end{array}$} & \multirow{2}{*}{$\begin{array}{l}\text { Cateter } \\
\text { Venoso } \\
\text { Central } \\
\end{array}$} & Neonatologia & 5.36 & 6.48 & 5.51 & 1.43 & 3.88 & 8.50 & 14.31 & P50 - P75 \\
\hline & & UCl Adultos & 7.81 & 3.04 & 2.26 & 0.00 & 0.59 & 2.06 & 4.96 & $>$ P90 \\
\hline & $\begin{array}{l}\text { Cateter } \\
\text { Venoso } \\
\text { Periférico }\end{array}$ & Neonatologia & 3.77 & 5.19 & 1.84 & 0.00 & 0.00 & 1.48 & 3.17 & > P90 \\
\hline \multirow{2}{*}{ Endometritis } & $\begin{array}{l}\text { Parto } \\
\text { Vaginal }\end{array}$ & G-O & 0.23 & 0.39 & 0.18 & 0.00 & 0.00 & 0.16 & 0.33 & P75 - P90 \\
\hline & $\begin{array}{c}\text { Parto } \\
\text { Cesarea }\end{array}$ & G-O & 0.44 & 0.79 & 0.42 & 0.00 & 0.09 & 0.32 & 0.66 & P75 - P90 \\
\hline $\begin{array}{l}\text { Infección } \\
\text { de Herida } \\
\text { Operatoria }\end{array}$ & $\begin{array}{l}\text { Parto } \\
\text { Cesarea }\end{array}$ & G-O & 1.55 & 1.6 & 1.2 & 0.17 & 0.67 & 1.41 & 2.19 & P75 - P90 \\
\hline $\begin{array}{l}\text { Infección del } \\
\text { Tracto Urinario }\end{array}$ & $\begin{array}{l}\text { Sonda } \\
\text { Vesical }\end{array}$ & UCI Adultos & 1.70 & 5.12 & 3.33 & 0.00 & 1.18 & 3.27 & 5.21 & P50 - P75 \\
\hline
\end{tabular}

Adaptado de: Quispe Z. Indicadores de la vigilancia epidemiológica de infecciones intrahospitalarias, 2013-2014; Boletín Epidemiológico CDC-MINSA, Perú. 24 (38): $764-767$.

intrahospitalaria en la unidad de cuidados intensivos materno (14.29 x 1000 d.d.e. comparado con $11.88 \times 1000$ d.d.e. a nivel nacional), la infección del torrente sanguíneo por catéter venoso central en la unidad de cuidados intensivos materno (7.81 x 1000 d.d.e. comparado con $2.26 \times 1000$ d.d.e. a nivel nacional), la infección del torrente sanguíneo por catéter venoso periférico en neonatología (3.77 x 1000 d.d.e comparado con 1.84 × 1000 d.d.e. a nivel nacional), la endometritis por parto vaginal $(0.23 \%$ comparado con $0.18 \%$ a nivel nacional), la endometritis por parto cesárea $(0.44 \%$ comparado con $0.42 \%$ a nivel nacional) y finalmente la infección de herida operatoria en cesárea (1.55\% comparado con $1.2 \%$ a nivel nacional). Esta comparación permite entender, que aunque nuestras tasas se mantienen menores en su mayoría cuando nos comparamos con los valores para establecimientos nivel III-2, aún tenemos que bajar nuestras tasas de incidencia para llegar a mejores niveles de atención en relación a este tipo de infecciones y compararnos con las tasas nacionales.

Sin embargo, existen dos tipos de infecciones que si ameritan nuestra atención cuando lo evaluamos de acuerdo a percentiles de medición; y estos son las infecciones del torrente sanguíneo por catéter venoso central en la unidad de cuidados intensivos materno y la infección del torrente sanguíneo por catéter venoso periférico en la unidad de cuidados intensivos e intermedios neonatales, que al valorar los resultados en percentiles ambas infecciones sobrepasan el P90; ello amerita acciones inmediatas de prevención en los servicios involucrados, sobre todo infección por catéter venoso central en la unidad de cuidados intensivos materno que además de evidenciar una tasa superior comparado con las tasas nacionales para hospitales nivel III-2, la tasa también se encuentra en más del P90 de lo permitido en los valores nacionales. Tabla 6.

Es importante entender que en la generación de las IAAS, intervienen diversos factores como por ejemplo, pacientes en condiciones de hacinamiento, pacientes con condiciones que permiten deficiencias de la inmunidad (edad, enfermedad, tratamientos, etc.), aparición de nuevos microorganismo, resistencia bacteriana, etc.; pero el factor más importante siempre será la posibilidad de que el personal de salud sea el que transporte microorganismos hacia el paciente por una deficiente higiene de manos o mal uso de los equipos de protección; las infecciones del torrente sanguíneo por catéter venoso central y catéter venoso periférico, la cual vemos en nuestra institución con tasas altas, tienen que ver mucho con este último punto y como institución debemos tomar medidas que permitan la corrección de estos problemas ${ }^{4}$.

Concluimos entonces que la prevalencia de IAAS en el Instituto Nacional Materno Perinatal teniendo como parámetro los egresos hospitalarios es 1.25 casos de infecciones por cada 100 pacientes que egresan de la institución. Las tasas de incidencia presentadas en la tabla $N^{\circ} 5$, serán mostrados como valores históricos y servirán como un parámetro de monitoreo para las evaluaciones sucesivas de casos de IAAS en nuestra institución. Al observar la tendencia a través de los años de las tasas de infecciones en el instituto y al compararlos con las tasas nacionales y las tasas según nivel de establecimiento de salud III-2, las infecciones del torrente sanguíneo por catéter venoso central en la unidad de cuidados intensivos materno y las infecciones del torrente sanguíneo por catéter venoso periférico en la unidad de cuidados intensivos e intermedios en el servicio de neonatología requieren 
acciones de control inmediatos. Aunque los valores en las tasas de neumonía por ventilación mecánica en la unidad de cuidados intensivos materno tiene valores altos que no sobrepasan las tasas promedios para hospitales nivel III-2, ni se encuentran en percentiles mayores del P90, la tasa observada en este último año de 25.95 por 1000 d.d.e. y la tendencia a aumentar en los últimos años nos permite decir que también tomemos acciones de control en este tipo de infección.

\section{REFERENCIAS BIBLIOGRÁFICAS}

1. Nodarse R.Visión Actualizada de las Infecciones Intrahospitalarias. Rev. Cubana Med. Milit. 2002; 31(3):201-8.

2. Horan TC, Andrus M, Dudeck MA. CDC/NHSN surveillance definition of health care-associated infection and criteria for specific types of infections in the acute care setting. American Journal of Infection Control. 2008;36(5):309-332. DOI: http:// dx.doi.org/10.1016/j.ajic.2008.03.002

3. Ministerio de Salud. Documento Técnico: Lineamientos para la Vigilancia, Prevención, y Control de las Infecciones Asociadas a la Atención de Salud.2015.

4. Ducel G, Fabry J, Nicolle L. Prevención de las infecciones nosocomiales: guía práctica. 2003. Disponible en: http:// apps.who.int/iris/handle/10665/67877.

5. Chincha O, Cornelio E, Valverde V, Acevedo M. "Infecciones Intrahospitalarias Asociadas a Dispositivos Invasivos en Unidades de Cuidados Intensivos de un Hospital Nacional de Lima, Perú". Rev Peruana Med Exp Salud Pública. 2013; 30(4):616-20.
6. Organización Panamericana de la Salud. Vigilancia epidemiológica de las infecciones asociadas a la atención de la salud. Modulo III Información para Gerentes y Directivos. OPS. Washington DC. 2012.

7. Organización Panamericana de la Salud. Una atención más limpia es una atención más segura. Disponible en: http:// www.who.int/gpsc/background/es/index.html

8. Villalobos AP, Barrero LI, Rivera SM, Ovalle MV, Valera D. Vigilancia de Infecciones Asociadas a la Atención en Salud, Resistencia Bacteriana y Consumo de Antibióticos en Hospitales de Alta Complejidad, Colombia, 2011. Biomédica. 2014; 34(1):67-80.

9. Diaz-Veliz C. Las Infecciones nosocomiales, un problema vigente. Rev Cuerpo Méd HNAAA 2016; 9(1):4-5.

10. Quispe Z. Indicadores de la vigilancia epidemiológica de infecciones intrahospitalarias, 2013-2014. Dirección General de Epidemiología, Ministerio de Salud. Bol Epidemiol (Lima). 2015;24(38):764-767. Disponible en: http://www.dge.gob.pe/ boletin.php

11. Instituto Nacional Materno Perinatal. Boletín Epidemiológico. 2015; 2.

12. Pujola M, Limónb E. Epidemiología general de las infecciones nosocomiales. Sistemas y programas de vigilancia. Enferm Infecc Microbiol Clin. 2013;31(2):108-113.

13. Quispe Z. Estudio Nacional de Prevalencia de IIH. Disponible en: $\quad$ www.minsa.gob.pe/dgsp/observatorio/.../1Estudio Nacional_Prevalencia_2015.pdf

\section{ORCID iDs}

Oswaldo M. Gonzales Carrillo https://orcid.org/0000-0001-9032-1542 